\title{
Investigation of the mechanism of the reduction of anthracycline-induced cardiotoxicity by Qishen Huanwu Capsule based on network pharmacology
}

\author{
Feng Wang ${ }^{1}$, Lixin Wang ${ }^{1}$, Fangru Liu ${ }^{2}$, Li Meng ${ }^{3}$, Na Zhao ${ }^{4}$, Xingrong Zhai ${ }^{5}$, Honggui Liu ${ }^{1}$, Jing Yang ${ }^{1}$ \\ ${ }^{1}$ Department of Cardiovascular Disease, Hebei Province Cangzhou Hospital of Integrated Traditional Chinese and Western Medicine, Cangzhou, \\ China; ${ }^{2}$ Department of Oral and Maxillofacial Surgery, Hebei Province Cangzhou Hospital of Integrated Traditional Chinese and Western Medicine, \\ Cangzhou, China; ${ }^{3}$ The Fourth Department of Internal Medicine, Yanshan County People's Hospital, Cangzhou, China; ${ }^{4}$ Department of Pediatric \\ Orthopedics, Hebei Province Cangzhou Hospital of Integrated Traditional Chinese and Western Medicine, Cangzhou, China; ${ }^{5}$ The Second \\ Department of Ultrasonic Diagnosis, Hebei Province Cangzhou Hospital of Integrated Traditional Chinese and Western Medicine, Cangzhou, \\ China \\ Contributions: (I) Conception and design: F Wang; (II) Administrative support: L Wang; (III) Provision of study materials or patients: F Wang, F \\ Liu; (IV) Collection and assembly of data: L Meng, N Zhao; (V) Data analysis and interpretation: F Wang, X Zhai, H Liu, J Yang; (VI) Manuscript \\ writing: All authors; (VII) Final approval of manuscript: All authors. \\ Correspondence to: Lixin Wang. Department of Cardiovascular Disease, Hebei Province Cangzhou Hospital of Integrated Traditional Chinese and \\ Western Medicine, Cangzhou, China. Email: wlx006@163.com.
}

Background: Cancer patients who receive anthracycline-based chemotherapy regimens often discontinue chemotherapy due to cardiotoxicity. Preventing and reducing anthracycline-induced cardiotoxicity (ACT) is a hot topic in cardio-oncology research. Network pharmacology is a new discipline that integrates pharmacology, bioinformatics, and systems biology. It can be used to analyze the mechanism of action of drugs in the body from a holistic perspective by constructing a "disease-gene-drug" network, providing a new method to explore compounding mechanisms of Chinese medicine. Based on network pharmacology, this study explored the mechanism of the reduction of cardiotoxicity of anthracyclines by Qishen Huanwu Capsule.

Methods: The active ingredients of Qishen Huanwu Capsule and their targets were screened based on the Traditional Chinese Medicine Systems Pharmacology Database and Analysis Platform and Chemistry Database. The target genes of ACT were screened through the PharmGkb, GeneCards, Online Mendelian Inheritance in Man (OMIM), Genetic Association Database (GAD), and Therapeutic Target Database (TTD). The Venny2.1 online analysis tool was used to construct a Venn diagram to obtain the common targets of ACT and Qishen Huanwu Capsule. The STRING platform was used to construct the proteinprotein interactions (PPI) among the common targets; ClueGO software was used to perform Gene Ontology (GO) biological process enrichment analysis for the common targets; the R language was used to perform Kyoto Encyclopedia of Genes and Genomes (KEGG) pathway enrichment analysis; and the results were visualized using Cytoscape software.

Results: The predictions indicate that Qishen Huanwu Capsule has 35 main active ingredients capable of reducing the cardiotoxicity of anthracyclines and that there are 36 common targets of ACT and Qishen Huanwu Capsule that are enriched in 133 biological processes and 27 signaling pathways.

Conclusions: Qishen Huanwu Capsule regulates phosphatidylinositol 3-kinase/protein kinase B (PI3K/ $\mathrm{Akt}$ ), mitogen-activated protein kinase (MAPK), forkhead box class $\mathrm{O}$ (FoxO) and other signaling pathways by regulating targets such as RAC-alpha serine/threonine protein kinase (Akt1), mitogen-activated protein kinase 1 (MAPK1), and mitogen-activated protein kinase 8 (MAPK8) and thereby inhibits oxidative stress and regulates apoptosis and autophagy to reduce the cardiotoxicity of anthracyclines.

Keywords: Qishen Huanwu Capsule; network pharmacology; anthracycline-induced cardiotoxicity (ACT); mechanism 
Submitted Aug 07, 2020. Accepted for publication Dec 01, 2020.

doi: 10.21037/apm-20-2204

View this article at: http://dx.doi.org/10.21037/apm-20-2204

\section{Introduction}

Cardiotoxicity is one of the most common side effects of anthracycline chemotherapeutics and can manifest as heart failure, coronary artery disease, heart valve disease, arrhythmia and other types of cardiovascular complications. Among these complications, the incidence rate for doxorubicin-induced left ventricular dysfunction is as high as $48 \%$ (1). Cardiotoxicity reduces cancer patient compliance with antitumor treatments and increases the mortality of cancer survivors. It has attracted increasingly more attention by medical experts. Preventing or reducing anthracycline-induced cardiotoxicity (ACT) is a hotspot in cardio-oncology research.

Qishen Huanwu Capsule (Ji Yao Zhi Zi Z20050798, batch number 030310) is an in-hospital formulation prepared by our hospital. It is derived from Buyang Huanwu Decoction and consists of Radix astragali (Huangqi), Radix Pseudostellariae (Taizishen), Semen Persicae (Taoren), Flos Carthami (Honghua), Radix Angelicae Sinensis (Danggui), Rhizoma Chuanxiong (Chuanxiong), Radix Paeoniae Rubra (Chishao), Radix Achyranthes Bidentatae (Niuxi), Rbizoma Pinelliae (Banxia) and other Chinese medicines. It can nourish Qi and Yin and remove blood stasis and phlegm. It is mainly used to treat stroke and coronary heart disease with Qi deficiency and blood stasis. The quercetin (2) and kaempferol (3) in Qishen Huanwu Capsule reduce the cardiotoxicity of anthracyclines. Based on network pharmacology, this study explored the main active ingredients, targets and pathways by which Qishen Huanwu Capsule reduces ACT, aiming to provide ideas and theoretical bases for the investigation of the specific mechanism of action by which Qishen Huanwu Capsule in reduces ACT.

We present the following article in accordance with the MDAR checklist (available at http://dx.doi.org/10.21037/ apm-20-2204).

\section{Methods}

\section{Medical ethics}

This study involved bioinformatics analysis only and thus did not require medical ethics approval. The study was conducted in accordance with the Declaration of Helsinki (as revised in 2013).

\section{Screening targets of the main components of Qishen Huanwu Capsule}

Based on the Traditional Chinese Medicine System Pharmacology Database and Analysis Platform (TCMSP, http://tcmspw.com/tcmsp.php) and Chemistry Database (http://www.organchem.csdb.cn/scdb/default.asp), we searched for all the active ingredients contained in Qishen Huanwu Capsule. Based on the pharmacokinetic parameters of the ingredients, oral bioavailability $(\mathrm{OB})$, drug-likeness (DL), Caco-2 cell permeability (Caco-2), and half-life (HL) were used as the parameters to screen the main active ingredients. The corresponding targets of the main active ingredients obtained by screening were searched in the TCMSP database and the Swiss Target Prediction database (http://swisstargetprediction.ch), and the UniProt database (http://www.uniprot.org/) was used to uniformly convert the target sites into gene names to construct a target library of the main active ingredients of Qishen Huanwu Capsule.

\section{Collection of ACT-related targets}

Using "anthracycline-induced cardiotoxicity (ACT)" as the query term, relevant targets were searched in the PharmGkb database (https://www.pharmgkb.org/), OMIM database (https://www.omim.org/), GeneCards database (https://www.genecards.org/), GAD database (https:// geneticassociationdb.nih.gov/), and TTD database (http:// db.idrblab.net/ttd/). After compilation, the target sites were used to construct an ACT-related target database.

\section{Venn analysis of Qishen Huanwu Capsule targets and the ACT target set}

Using the Venny 2.1 online analysis tool (https://bioinfogp. cnb.csic.es/tools/venny/Index.html), we merged the main active ingredient target library of Qishen Huanwu Capsule and the ACT target data set to obtain a common gene set and explored the target sites through which Qishen Huanwu Capsule acts to treat ACT. 
Construction and analysis of a protein-protein interaction (PPI) network

The common targets of Qishen Huanwu Capsule and ACT obtained by Venn analysis were input into the STRING (https://string-db.org/) platform to construct a common target PPI network; the organism was set to "Homo sapiens", and the minimum required interaction score was set to "high confidence (0.7)". We used R language software to determine the occurrence frequency and draw a histogram. All R packages can be downloaded through R (https://www.r-project.org/) and Bioconductor (https:// www.bioconductor.org/).

\section{Gene Ontology (GO) function enrichment of key target genes and Kyoto Encyclopedia of Genes and Genomes (KEGG) pathway analysis}

We used the ClueGO plug-in of Cytoscape 3.2.1 software to perform GO biological process enrichment analysis on the common targets of Qishen Huanwu Capsule and ACT obtained by Venn analysis; the analysis results were presented as a pie chart. $R$ language software was used to perform KEGG pathway enrichment analysis on the common targets, and the analysis results were presented in the form of bubble diagrams. Cytoscape 3.2.1 software was used to construct a componenttarget-pathway network to analyze the mechanism of action by which Qishen Huanwu Capsule reduces ACT.

\section{Results}

\section{Screening of the main active ingredients of Qishen Huanwu Capsule}

Qishen Huanwu Capsule comprises Chinese medicines, such as Huangqi, Taizishen, Taoren, Honghua, Danggui, Chuanxiong, Chishao, Niuxi and Banxia. Using the TCMSP database and Chemistry Database and based on $\mathrm{OB} \geq 30 \%$, DL $\geq 0.18$, Caco- $2 \geq-0.04$, and HL $\geq 4$ as pharmacokinetic parameters (4), 10 active ingredients of Huangqi, 3 active ingredients of Taizishen, 7 active ingredients of Taoren, 10 active ingredients of Honghua, 2 active ingredients of Danggui, 4 active ingredients of Chuanxiong, 4 active ingredients of Chishao, 9 active ingredients of Niuxi, and 4 active ingredients of Banxia were identified. Duplicated ingredients were removed, resulting in a total of 35 main active ingredients, e.g., quercetin, luteolin, kaempferol, hederagenin and beta-sitosterol (Table 1).

\section{Target prediction}

Using the TCMSP database and the Swiss Target Prediction database, we searched the targets of the active ingredients contained in Qishen Huanwu Capsule and retrieved 1,848 targets. After duplicate targets were removed, we obtained a total of 230 targets.

For ACT targets, we used "anthracycline-induced cardiotoxicity" as the search term and retrieved 146 ACTrelated targets: 122 in the GeneCards database, 18 in the PharmGkb database, 3 in the OMIM database, and 3 in the GAD database.

We input 230 Qishen Huanwu Capsule targets and 146 ACT targets into Venny 2.1 software to generate a Venn diagram. A total of 36 targets common to both Qishen Huanwu Capsule and ACT were obtained from the intersection of the 2 sets, as shown in Figure 1 and Table 2. These 36 targets could be the potential targets of Qishen Huanwu Capsule, leading to a reduction in ACT.

\section{Diagram showing the network relationship between the active ingredients of Qishen Huanwu Capsule and ACT- related targets}

We input the active ingredients of Qishen Huanwu Capsule and the potential targets for ACT treatment into Cytoscape 3.2.1 software to establish a network diagram with close connections between nodes, as shown in Figure 2. The network has 71 nodes and 210 edges. The blue circle represents the main active ingredients of Qishen Huanwu Capsule, and the green circle represents the potential targets of Qishen Huanwu Capsule for the treatment of ACT. The degree of a node in the network refers to the number of edges associated with the node; a higher degree indicates that the compound is associated with more targets. In this network, the average degree of the active ingredients of Qishen Huanwu Capsule is 5.27, and the top 5 compounds in terms of degree are quercetin (degree $=30$ ), luteolin (degree $=15)$, kaempferol (degree $=14), \beta$-carotene (degree $=12$ ), and baicalein (degree $=11$ ), suggesting that these compounds are the key active ingredients in Qishen Huanwu Capsule for the treatment of ACT.

\section{Analysis of the target PPI network}

The 36 potential targets obtained from the above screening were imported into the STRING database to obtain a PPI 
Table 1 Qishen Huanwu Capsule: main active ingredients and their pharmacokinetic parameters

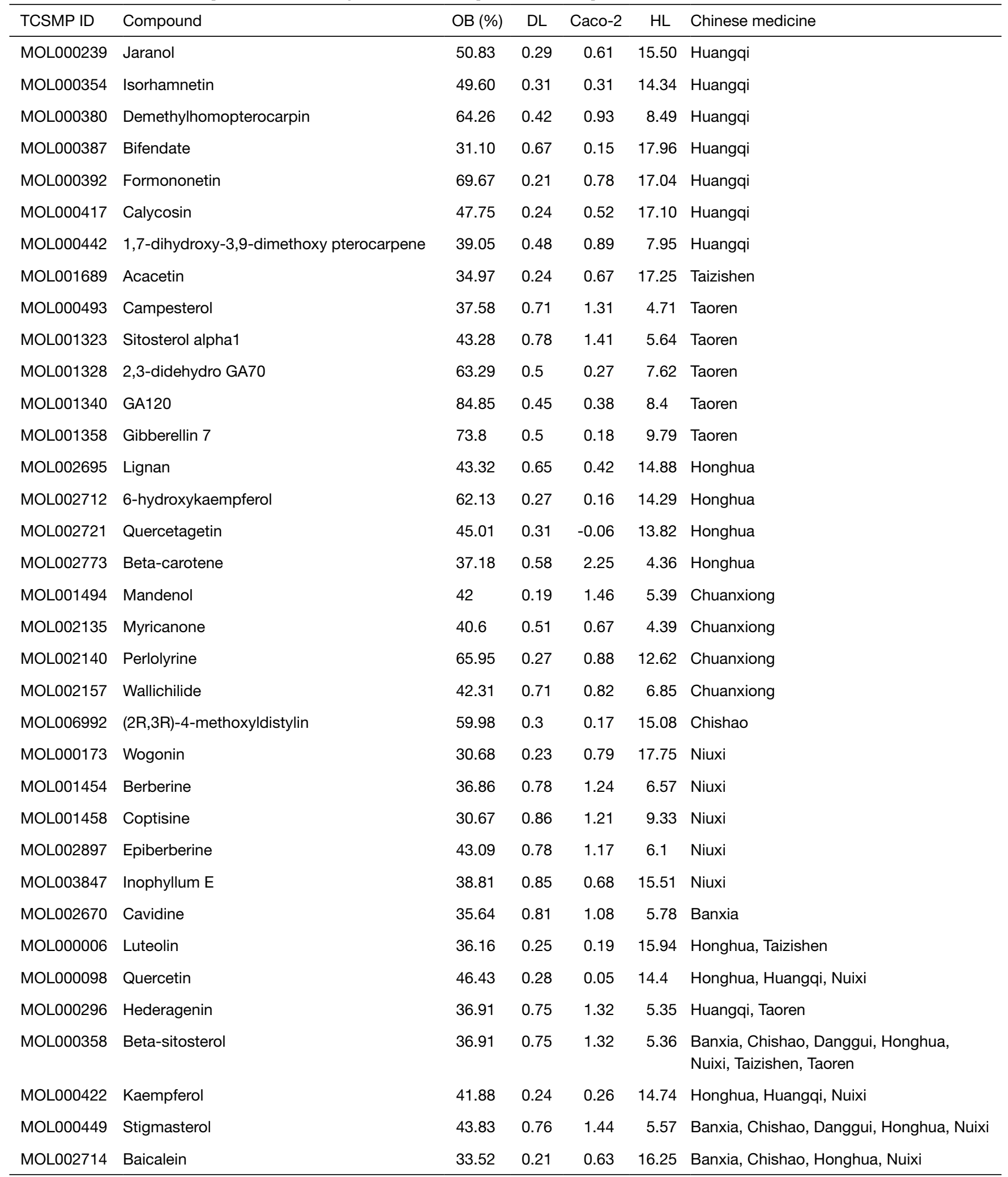

$\mathrm{OB}$, oral bioavailability; DL, drug-likeness; Caco-2, Caco-2 cell permeability; HL, half-life. 


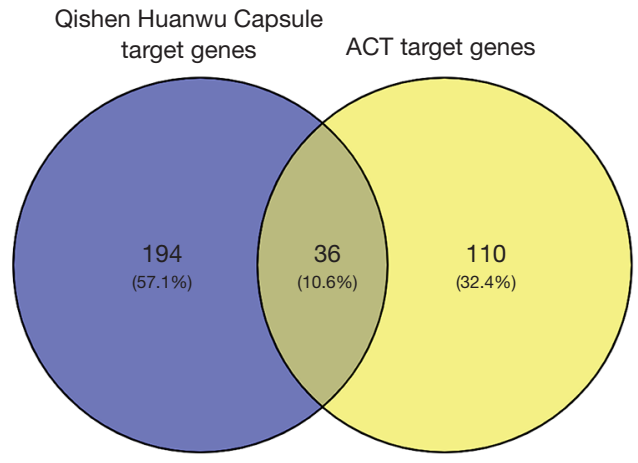

Figure 1 The Venn diagram of Qishen Huanwu Capsule targets and ACT-related targets. ACT, anthracycline-induced cardiotoxicity.

Table 2 Targets common to both Qishen Huanwu Capsule and ACT

\begin{tabular}{lclc}
\hline Gene ID & $\begin{array}{l}\text { Gene } \\
\text { name }\end{array}$ & Name of encoded protein & $\begin{array}{c}\text { Degree } \\
\text { value }\end{array}$ \\
\hline Q96B36 & AKT1 & $\begin{array}{l}\text { RAC-alpha serine/threonine } \\
\text { protein kinase }\end{array}$ & 34 \\
P45983 & MAPK8 & Mitogen-activated protein kinase 8 & 31 \\
P28482 & MAPK1 & Mitogen-activated protein kinase 1 & 31 \\
P42574 & CASP3 & Caspase-3 & 30 \\
P01106 & MYC & Myc proto-oncogene protein & 30 \\
P35354 & PTGS2 & Prostaglandin G/H synthase 2 & 29 \\
P15692 & VEGFA & Vascular endothelial growth & 29 \\
& & factor A & 26 \\
P01133 & EGF & Pro-epidermal growth factor & 25 \\
Q8MLW0 & EGFR & Receptor protein-tyrosine kinase & 25 \\
P07900 & HSP90AA1 & Heat shock protein HSP 90-alpha & 24 \\
P29474 & NOS3 & Nitric oxide synthase & 24 \\
P01100 & FOS & Proto-oncogene c-Fos & 23 \\
Q16665 & HIF1A & Hypoxia-inducible factor 1-alpha & 22 \\
P55211 & CASP9 & Caspase-9 & 22 \\
\hline
\end{tabular}

Table 2 (continued)

network, and the PPI network was further analyzed using the cytoHubba plug-in in Cytoscape 3.2.1 software. The results are shown in Figure 3. $\mathrm{R}$ was used to determine the top 30 targets in regard to frequency, resulting in the bar graph shown in Figure 4. The network in Figure 3 contains
Table 2 (continued)

\begin{tabular}{|c|c|c|c|}
\hline Gene ID & $\begin{array}{l}\text { Gene } \\
\text { name }\end{array}$ & Name of encoded protein & $\begin{array}{l}\text { Degree } \\
\text { value }\end{array}$ \\
\hline Q16539 & MAPK14 & $\begin{array}{l}\text { Mitogen-activated protein kinase } \\
14\end{array}$ & 22 \\
\hline P09601 & HMOX1 & Heme oxygenase 1 & 22 \\
\hline P04626 & ERBB2 & $\begin{array}{l}\text { Receptor tyrosine-protein kinase } \\
\text { erbb-2 }\end{array}$ & 22 \\
\hline P35222 & CTNNB1 & Catenin beta-1 & 21 \\
\hline Q07820 & MCL1 & $\begin{array}{l}\text { Induced myeloid leukemia cell } \\
\text { differentiation protein } \mathrm{Mcl}-1\end{array}$ & 20 \\
\hline P00441 & SOD1 & Superoxide dismutase (Cu-Zn) & 19 \\
\hline Q16236 & NFE2L2 & $\begin{array}{l}\text { Nuclear factor erythroid 2-related } \\
\text { factor } 2\end{array}$ & 19 \\
\hline Q00987 & MDM2 & e3 ubiquitin-protein ligase Mdm2 & 18 \\
\hline P05164 & MPO & Myeloperoxidase & 16 \\
\hline Q03135 & CAV1 & Caveolin-1 & 16 \\
\hline P17302 & GJA1 & Gap junction alpha-1 protein & 15 \\
\hline P48023 & FASLG & $\begin{array}{l}\text { Tumor necrosis factor ligand } \\
\text { superfamily member } 6\end{array}$ & 15 \\
\hline P15559 & NQO1 & $\begin{array}{l}\mathrm{NAD}(\mathrm{P}) \mathrm{H} \text { dehydrogenase } \\
\text { (quinone) } 1\end{array}$ & 14 \\
\hline P09211 & GSTP1 & Glutathione S-transferase P & 13 \\
\hline P10415 & $B C L 2$ & Apoptosis regulator $\mathrm{Bcl}-2$ & 12 \\
\hline Q07812 & $B A X$ & Apoptosis regulator BAX & 11 \\
\hline Q9UNQ0 & $A B C G 2$ & $\begin{array}{l}\text { Broad substrate specificity } \\
\text { ATP-binding cassette transporter } \\
\text { ABCG2 }\end{array}$ & 11 \\
\hline P16581 & SELE & E-selectin & 10 \\
\hline Q07869 & PPARA & $\begin{array}{l}\text { Peroxisome proliferator-activated } \\
\text { receptor alpha }\end{array}$ & 7 \\
\hline Q00613 & HSF1 & Heat shock factor protein 1 & 7 \\
\hline Q01320 & TOP2A & DNA topoisomerase 2-alpha & 6 \\
\hline P78380 & OLR1 & $\begin{array}{l}\text { Oxidized low-density lipoprotein } \\
\text { receptor } 1\end{array}$ & 4 \\
\hline
\end{tabular}

ACT, anthracycline-induced cardiotoxicity.

36 nodes and 350 edges, with an average node degree of 19.4; the transition from yellow to red indicates the gradual increase in node degree. The targets with higher protein interaction frequencies are Akt1, MAPK1, and MAPK8, suggesting that these targets may be the key targets of 


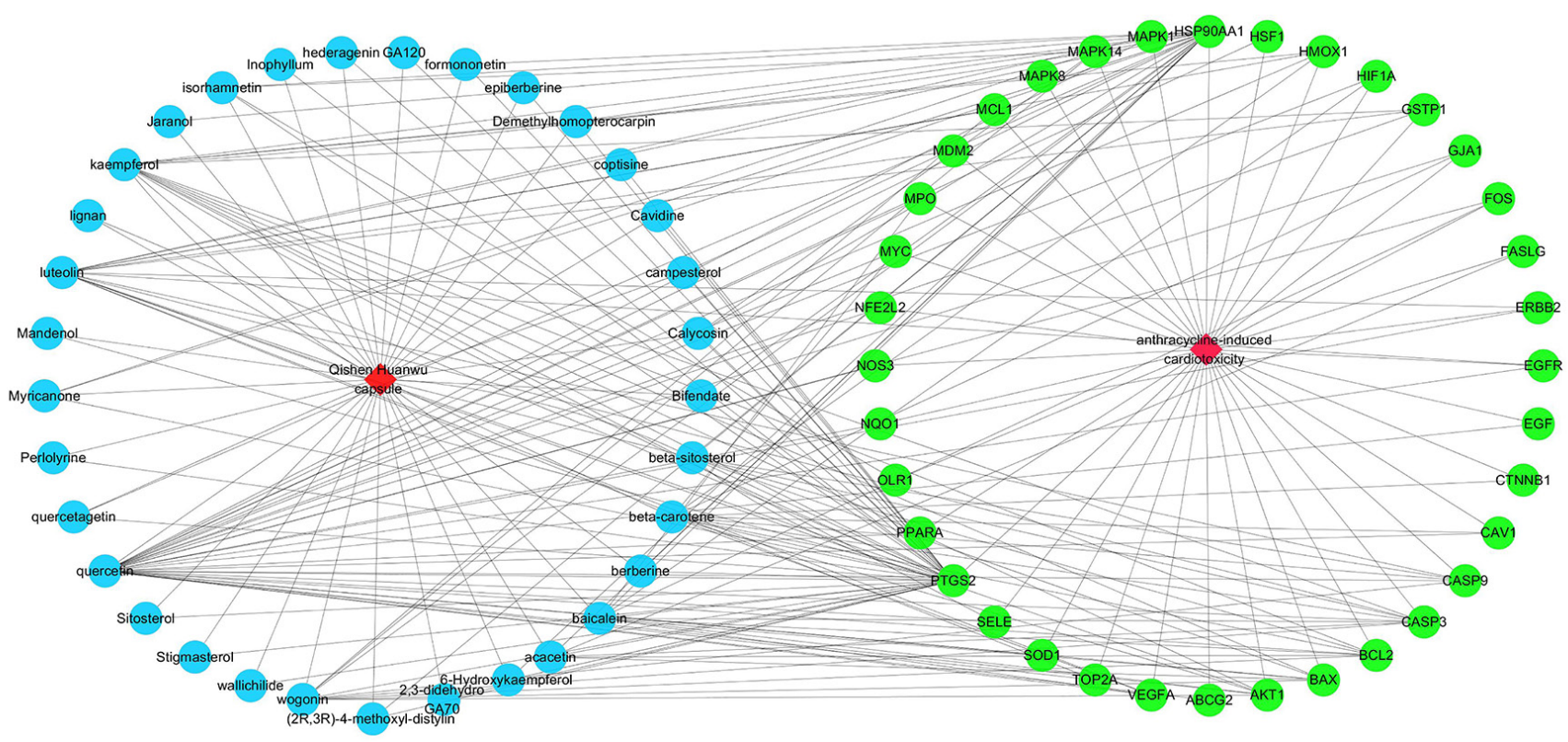

Figure 2 The network diagram for Qishen Huanwu Capsule targets and ACT-related targets. ACT, anthracycline-induced cardiotoxicity.

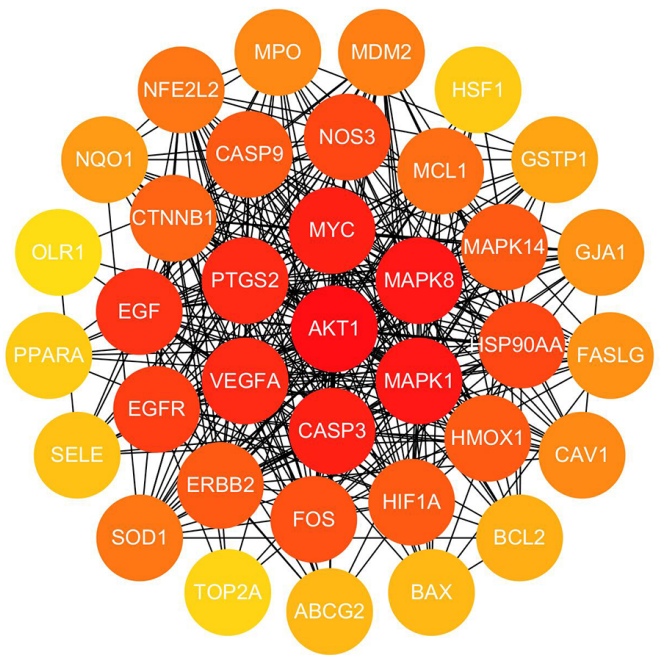

Figure 3 Common target protein interaction network.

Qishen Huanwu Capsule in the treatment of ACT.

\section{GO biological process analysis and KEGG patbway enrichment analysis}

We used the ClueGo plug-in to perform GO biological process analysis of 36 common targets, resulting in the pie chart shown in Figure 5. As seen in the figure, the common

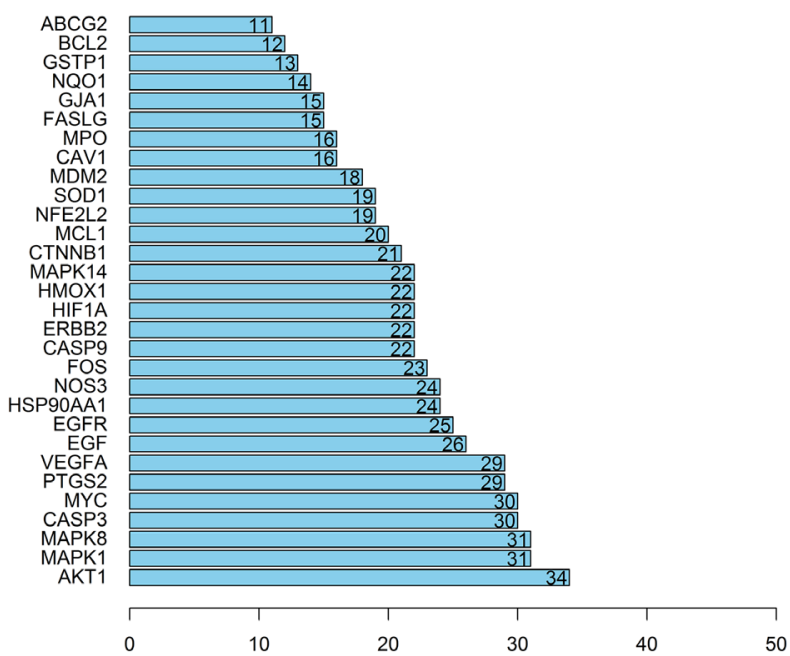

Figure 4 Frequencies of common target proteins.

targets are mainly concentrated in 133 biological processes, including negative regulation of the apoptotic signaling pathway, regulation of reactive oxygen species metabolic processes, and regulation of DNA binding, suggesting that Qishen Huanwu Capsule can reduce the cardiotoxicity of anthracycline chemotherapeutics through mechanisms such as regulating apoptosis, oxidative stress, and DNA damage repair. $\mathrm{R}$ was used to perform KEGG pathway 


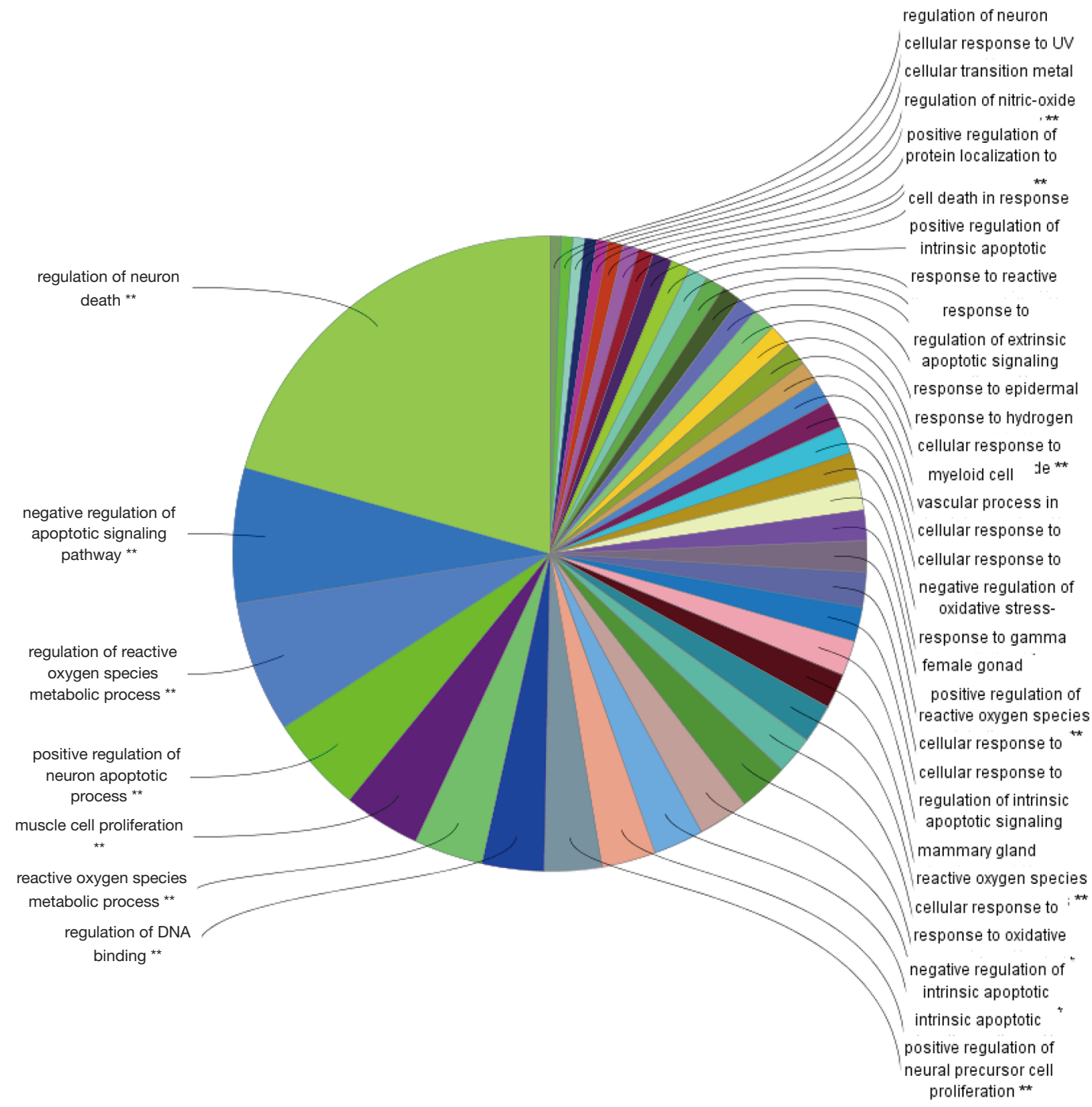

Figure 5 Biological process pie chart (ClueGo functional analysis).

enrichment analysis on the common targets. Common targets were mainly enriched in 27 signaling pathways, such as the phosphatidylinositol 3-kinase/protein kinase $\mathrm{B}$ (PI3K/Akt) signaling pathway, the mitogen-activated protein kinase (MAPK) signaling pathway, and the hypoxiainducible factor-1 (HIF-1) signaling pathway. The top 20 pathways are shown in Table 3 and Figure 6. The main active ingredients of Qishen Huanwu Capsule, the common targets of Qishen Huanwu Capsule and ACT, and KEGG pathway analysis were used to construct a componenttarget-pathway network diagram (Figure 7). As shown in Figure 8, a total of 14 genes (HSP90AA1, AKT1, VEGFA,
BCL2, CASP9, MYC, EGFR, MAPK1, MDM2, ERBB2, MCL1, EGF, NOS3, and FASLG) were enriched in the PI3K/Akt signaling pathway, suggesting that Qishen Huanwu Capsule can reduce ACT through multiple targets, multiple pathways, and multiple mechanisms.

\section{Discussion}

Anthracycline chemotherapeutics, represented by doxorubicin, play an important role in the treatment of various cancers. Unfortunately, side effects such as cardiotoxicity have severely restricted the clinical 
Table 3 Target pathway enrichment results (top 20)

\begin{tabular}{|c|c|c|c|}
\hline ID & Name & Number of targets & $P$ value \\
\hline hsa01522 & Endocrine resistance & $10 / 35$ & $1.69 \mathrm{E}-10$ \\
\hline hsa04066 & HIF-1 signaling pathway & $10 / 35$ & $4.92 E-10$ \\
\hline hsa04210 & Apoptosis & $10 / 35$ & $4.40 \mathrm{E}-09$ \\
\hline hsa01521 & EGFR tyrosine kinase inhibitor resistance & $8 / 35$ & $1.56 \mathrm{E}-08$ \\
\hline hsa04370 & VEGF signaling pathway & $7 / 35$ & $4.50 \mathrm{E}-08$ \\
\hline hsa04010 & MAPK signaling pathway & $12 / 35$ & $7.75 \mathrm{E}-08$ \\
\hline hsa04510 & Focal adhesion & $10 / 35$ & $1.71 \mathrm{E}-07$ \\
\hline hsa04215 & Apoptosis-multiple species & $5 / 35$ & $1.12 \mathrm{E}-06$ \\
\hline hsa04071 & Sphingolipid signaling pathway & $7 / 35$ & $5.70 \mathrm{E}-06$ \\
\hline hsa04115 & p53 signaling pathway & $5 / 35$ & $6.51 \mathrm{E}-05$ \\
\hline hsa04015 & Rap1 signaling pathway & $7 / 35$ & 0.000222 \\
\hline hsa04014 & Ras signaling pathway & $7 / 35$ & 0.000408 \\
\hline hsa04520 & Adherens junction & $4 / 35$ & 0.00084 \\
\hline hsa04380 & Osteoclast differentiation & $5 / 35$ & 0.000964 \\
\hline hsa04550 & Signaling pathways regulating pluripotency of stem cells & $3 / 35$ & 0.001186 \\
\hline
\end{tabular}

application of these drugs. The exact mechanism of ACT is still unclear. Current research shows that the molecular mechanism involves multiple aspects such as oxidative stress, apoptosis, autophagy, and type II topoisomerase-related DNA damage (5). Traditional Chinese medicine believes that chemotherapy drugs are heat toxins. Heat toxins are stored in the body and thereby decoct body fluid, consume Qi and injure Yin, resulting in Qi and Yin deficiency. Qi deficiency leads to a lack of strength, resulting in blood stasis, and heat toxins can produce phlegm from body fluid. Therefore, frequent blood and phlegm stasis, Qi and Yin deficiency, and the mutual accumulation of phlegm and blood stasis are the main symptoms of ACT. Qishen Huanwu Capsule is derived from Buyang Huanwu Decoction, in which Huangqi, as the primary active compound, nourishes vitality (Qi vitality facilitates blood circulation); Taizishen, as an important secondary compound, nourishes Qi, promotes body fluid and blood movement, helps Huangqi replenish Qi, and nourishes Yin; Danggui nourishes the blood and promotes blood circulation; Taoren, Honghua, Chishao, Chuanxiong, and Niuxi promote blood circulation and remove blood stasis; and Banxia loosens phlegm and reduces thirst. The whole prescription nourishes Qi and Yin and removes blood stasis and phlegm. Its mechanism and formulation target the main symptoms of ACT.

The PPI network shows that the main active ingredients of Qishen Huanwu Capsule may act through targets it has in common with ACT, such as Akt1, MAPK1, and MAPK8. Akt is a serine/threonine protein kinase and an important downstream target kinase in the PI3 K signal transduction pathway. Akt1 is a Akt subtype highly expressed in myocardial tissues, and it has important effects on the growth/hypertrophy, survival/apoptosis, and metabolism of cardiomyocytes. Akt downregulates downstream forkhead box class $\mathrm{O} 3 \mathrm{a}$ (FoxO3a) and inhibits oxidative stress through the Akt/FoxO3a pathway (6); it 


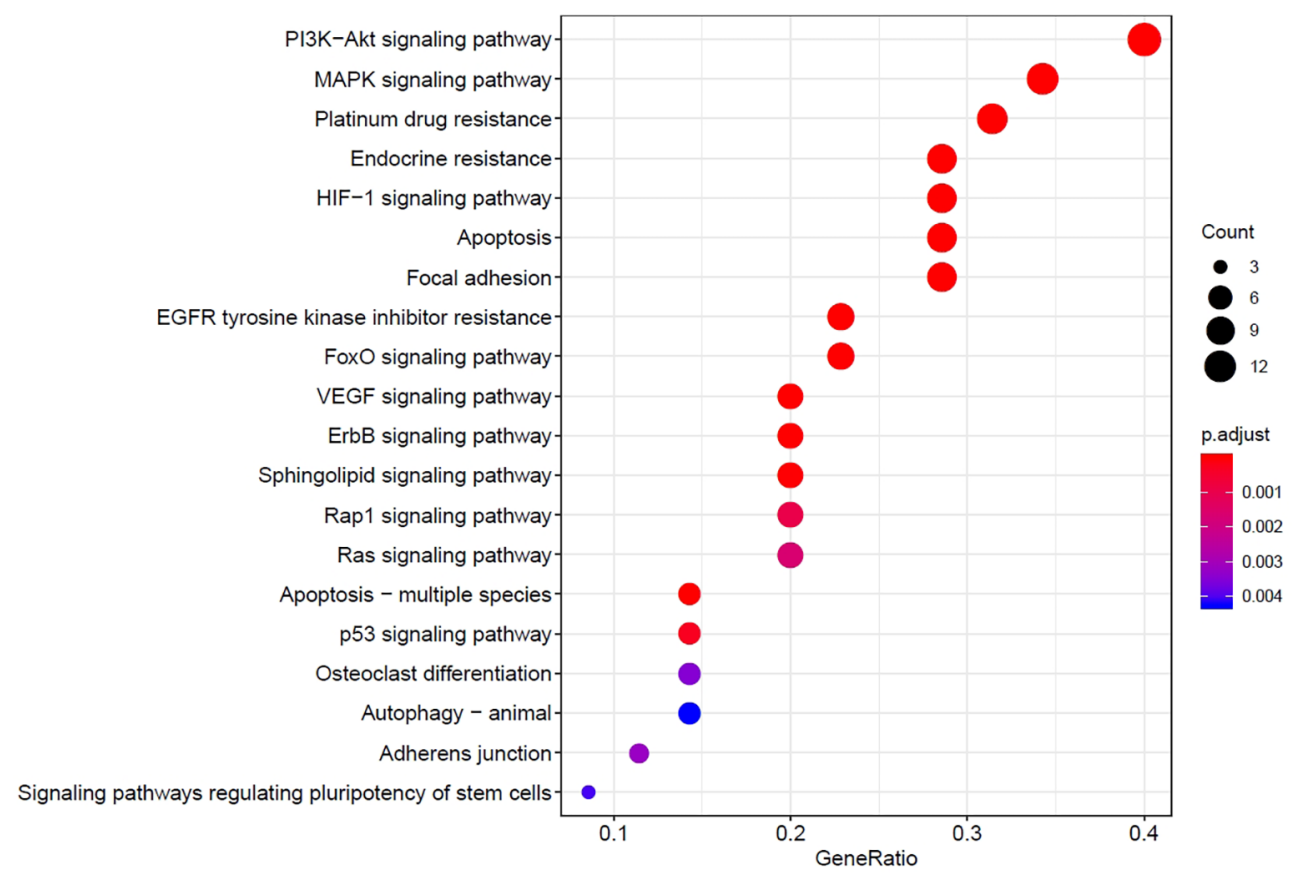

Figure 6 KEGG pathway enrichment (top 20).

acts through the Akt/glycogen synthase kinase-3 $\beta$ pathway, upregulates the expression of B-cell lymphoma 2 (Bcl-2), downregulates the expression of caspase- 3 , and exerts an anti-apoptotic effect (7). Mitogen-activated protein kinases (MAPKs) are a group of serine/threonine protein kinases that can sense extracellular stimuli and trigger a wide range of intracellular responses. Activated MAPKs phosphorylate a variety of target proteins, including transcription factors, such as c-Jun, c-Myc, and activating transcription factor 2, and apoptosis-related proteins, such as Bcl-2 and Bcl-2associated death promoter, and thus regulate proliferation, differentiation, apoptosis, oxidative stress, inflammation and other cellular activities (8). Doxorubicin, an anthracycline, can activate the MAPK signaling pathway, promote the phosphorylation of apoptotic proteins, induce oxidative stress, and cause myocardial damage (9). It can also degrade inhibitor $\kappa \mathrm{B} \alpha$ protein through the MAPK/nuclear factor kappa B signaling pathway, leading to the release of inflammatory mediators and subsequent cardiomyocyte apoptosis (10). Regulating the PI3K/Akt and MAPK signal pathways through Akt1, MAPK1, MAPK8 and other targets is a potential mechanism of action of Qishen Huanwu Capsule in treating ACT.

Oxidative stress is caused by the unbalanced response of reactive oxygen species and endogenous antioxidants to injury, and it is one of the main mechanisms of cardiotoxicity caused by anthracycline chemotherapeutics. Quercetin, kaempferol, isorhamnetin and other ingredients in Qishen Huanwu Capsule can reduce ACT by inhibiting oxidative stress. Quercetin upregulates the expression of 14-3-3 $\gamma$, increases the level of superoxide dismutase (SOD) and glutathione peroxidase in cardiomyocytes, reduces the level of malondialdehyde (MDA) and reactive oxygen species, inhibits oxidative stress, improves mitochondrial function, and reduces doxorubicin-induced damage to cardiomyocytes (2). Kaempferol binds to the promoter region of the proapoptotic gene $\mathrm{Bcl}-2$-associated $\mathrm{X}$ protein (Bax), inhibits the p53 signaling pathway and the extracellular signalregulated kinase-dependent MAPK pathway activated by doxorubicin, and reduces the oxidative stress, apoptosis and injury, and mitochondrial dysfunction caused by doxorubicin (3). Isorhamnetin increases SOD, catalase and glutathione peroxidase activity through the MAPK signaling pathway, reduces MDA activity, and reduces the oxidative stress caused by doxorubicin (11). The PI3K/Akt, MAPK, P53 and other signaling pathways were all enriched with the abovementioned active ingredients, as determined by KEGG analysis.

Anthracyclines can cause myocardial damage by inducing cardiomyocyte apoptosis. The expression and 


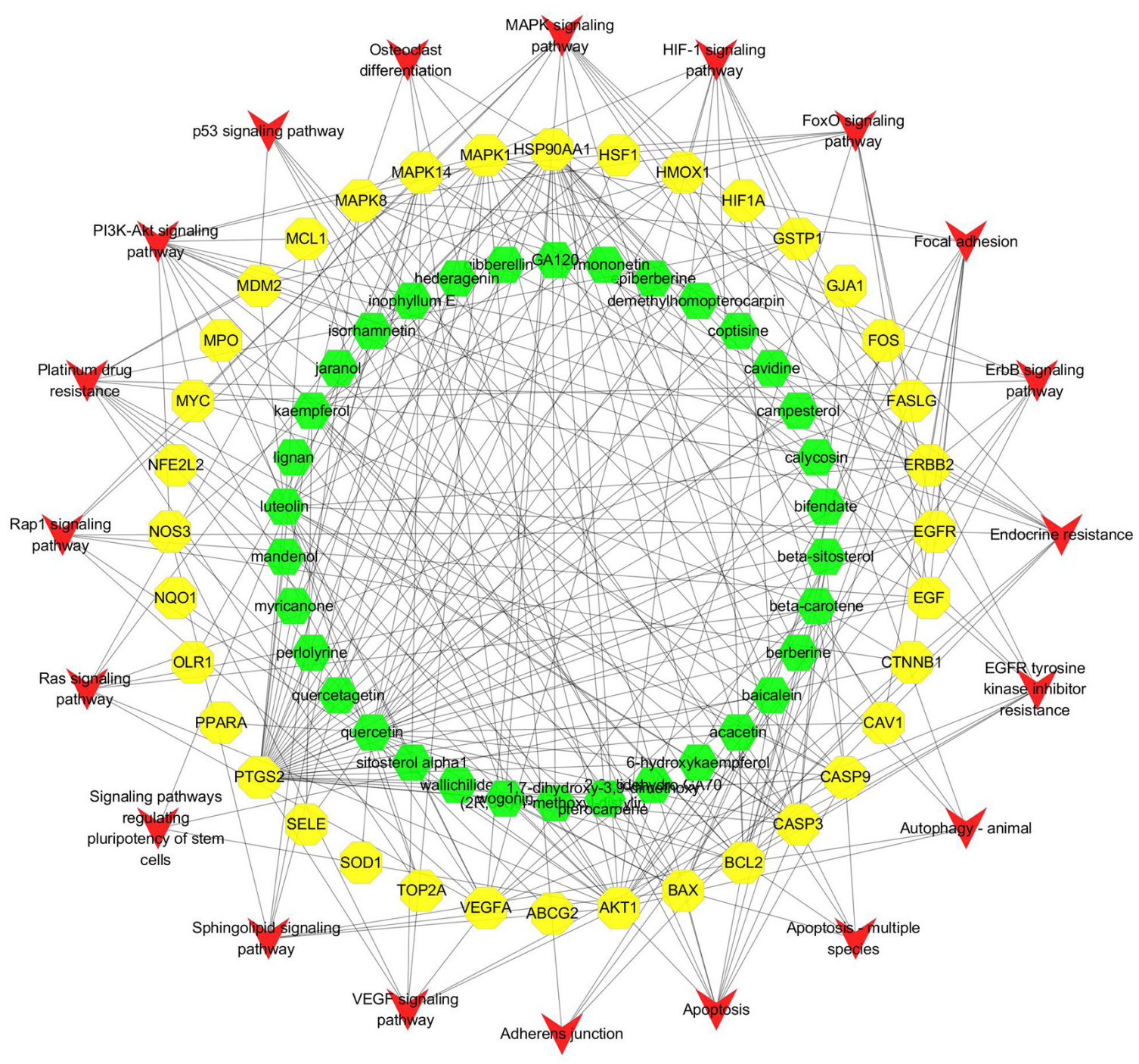

Figure 7 Component-target-pathway network.

regulation of the Bcl-2 family are key factors of apoptosis. $\mathrm{Bcl}-2$ is an apoptosis inhibitor protein, and Bax is a proapoptotic protein. These proteins regulate apoptosis through the PI3K/Akt pathway and P53 pathway (12). In Qishen Huanwu Capsule, quercetin, kaempferol, baicalein, $\beta$-carotene, $\beta$-sitosterol, wogonin, and acacetin act on the Bcl-2 family. Baicalein antagonizes cardiotoxicity caused by doxorubicin by upregulating the expression of Bcl-2, downregulating the expression of Bax, reducing the ratio of Bax/Bcl-2, and inhibiting cardiomyocyte apoptosis (13). Quercetin and $\beta$-sitosterol directly or indirectly regulate CASP9 and CASP 3 in the CASP cascade reaction, which may be an important mechanism by which Qishen Huanwu
Capsule regulates apoptosis (14). Luteolin downregulates the expression of p-phosphatase and tensin homolog deleted on chromosome ten (PTEN), p-Akt, p-extracellular signal-regulated kinase, p-mammalian target of rapamycin (mTOR), and p-glycogen synthase kinase-3 $\beta$ in $\mathrm{H} 9 \mathrm{C} 2$ rat cardiomyocytes and reduces $\mathrm{H} 9 \mathrm{C} 2$ cell apoptosis induced by doxorubicin through the PTEN/Akt and extracellular signal-regulated kinase pathways (15).

Autophagy plays an important role in cardiotoxicity induced by anthracyclines (16). Autophagy is a type of protein degradation pathway that depends on lysosomes. It mainly acts to degrade damaged organelles and misfolded proteins in cells and is widely involved in a 


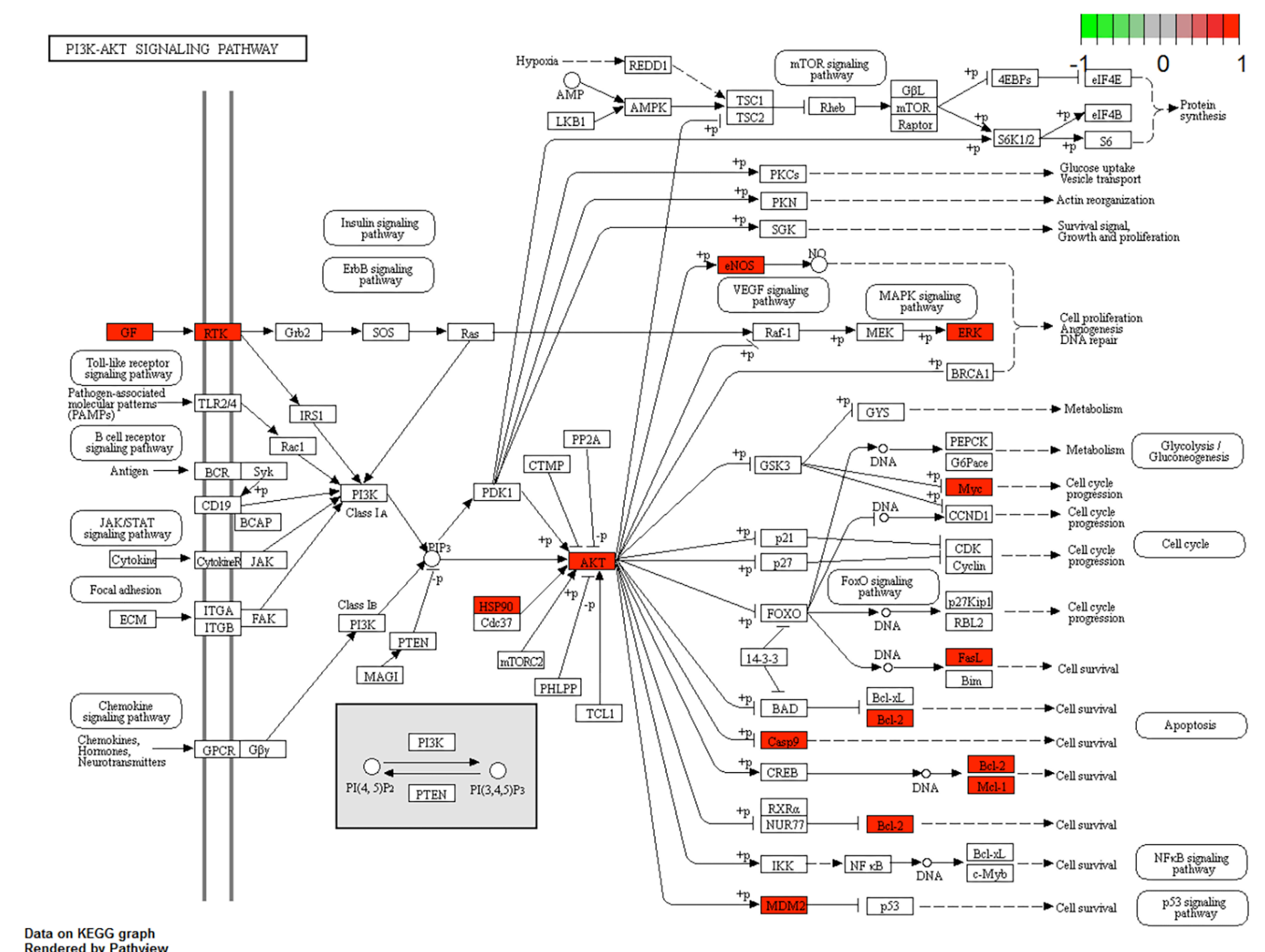

Figure $8 \mathrm{PI}$ K/Akt signaling pathway.

variety of physiological and pathological processes (17). The basal level of autophagy is of great significance for maintaining homeostasis, whereas overactivated autophagy causes cell death due to the excessive degradation of intracellular components (18). The PI3K/Akt pathway activates the downstream effector molecule mTOR to

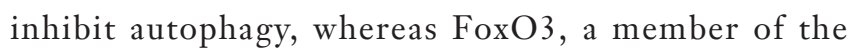
FoxO family, activates autophagy by upregulating Atg or autophagy-regulatory genes. HIF- $1 \alpha$ upregulates the expression of BCL2-interacting protein 3 and Beclin-1 to transform microtubule-associated protein light chain 3 (LC3)-I into LC3-II, thus inducing autophagy (19). In this study, based on KEGG analysis, the PI3K/Akt, FoxO and HIF-1 pathways that regulate autophagy were all enriched. In addition, the main active ingredient of Qishen Huanwu Capsule, baicalein, upregulates the expression of membrane-associated RING-CH 5 in cardiomyocytes through the KLF4-MARCH5-Drp1 pathway, inhibits mitochondrial division caused by $\mathrm{H}_{2} \mathrm{O}_{2}$ and ischemiareperfusion, enhances mitochondrial autophagy and reduces cardiomyocyte apoptosis caused by anthracyclines (20). These results suggest that Qishen Huanwu Capsule can reduce ACT by regulating autophagy.

This study systematically predicted, through network pharmacology, the potential mechanism by which Qishen Huanwu Capsule reduces ACT. We analyzed and constructed the "Active Ingredients of Qishen Huanwu Capsule-ACT-related targets" network diagram for Qishen Huanwu Capsule-mediated reduction in ACT and identified 35 main active ingredients of Qishen Huanwu Capsule and 36 targets common to both Qishen Huanwu Capsule and ACT. These results reflect the possible mechanism by 
which Qishen Huanwu Capsule reduces ACT and provide ideas and theoretical bases for further verification of its pharmacological mechanism.

\section{Acknowledgments}

Funding: Traditional Chinese Medicine Scientific Research Project of Hebei Provincial Administration of Traditional Chinese Medicine (Project Number: 2020502).

\section{Footnote}

Reporting Checklist: The authors have completed the MDAR checklist. Available at http://dx.doi.org/10.21037/apm-202204

Conflicts of Interest: All authors have completed the ICMJE uniform disclosure form (available at http://dx.doi. org/10.21037/apm-20-2204). The authors have no conflicts of interest to declare.

Ethical Statement: The authors are accountable for all aspects of the work in ensuring that questions related to the accuracy or integrity of any part of the work are appropriately investigated and resolved. This study involved bioinformatics analysis only and thus did not require medical ethics approval. The study was conducted in accordance with the Declaration of Helsinki (as revised in 2013).

Open Access Statement: This is an Open Access article distributed in accordance with the Creative Commons Attribution-NonCommercial-NoDerivs 4.0 International License (CC BY-NC-ND 4.0), which permits the noncommercial replication and distribution of the article with the strict proviso that no changes or edits are made and the original work is properly cited (including links to both the formal publication through the relevant DOI and the license). See: https://creativecommons.org/licenses/by-nc-nd/4.0/.

\section{References}

1. Zamorano JL, Lancellotti P, Rodriguez Muñoz D, et al. 2016 ESC position paper on cancer treatments and cardiovascular toxicity developed under the auspices of the ESC committee for practice guidelines. Eur Heart J 2016;37:2768-801.

2. Chen X, Peng X, Luo Y, et al. Quercetin protects cardiomyocytes against doxorubicin-induced toxicity by suppressing oxidative stress and improving mitochondrial function via 14-3-3 $\gamma$. Toxicol Mech Methods 2019;29:344-54.

3. Xiao J, Sun GB, Sun B, et al. Kaempferol protects against doxorubicin-induced cardiotoxicity in vivo and in vitro. Toxicology 2012;292:53-62.

4. Zhao M, Chen Y, Wang C, et al. Systems Pharmacology Dissection of Multi-Scale Mechanisms of Action of Huo-Xiang-Zheng-Qi Formula for the Treatment of Gastrointestinal Diseases. Front Pharmacol 2019;9:1448.

5. Dos Santos Arruda F, Tomé FD, Miguel MP, et al. Doxorubicin-Induced Cardiotoxicity and Cardioprotective Agents: Classic and New Players in the Game. Curr Pharm Des 2019;25:109-18.

6. Xue J,Zhang X,Bian W, et al. Alleviation of doxorubicininduced cardiotoxicity by Hong Huang decoction may involve a reduction in myocardial oxidative stress and activation of Akt/FoxO3a pathways. Int J Clin Exp Med 2018;11:10574-84.

7. Abbas NAT, Kabil SL. Liraglutide ameliorates cardiotoxicity induced by doxorubicin in rats through the Akt/GSK-3 $\beta$ signaling pathway.Naunyn Schmiedebergs Arch Pharmacol 2017;390:1145-53.

8. Kim EK, Choi EJ. Compromised MAPK signaling in human diseases: an update. Arch Toxicol 2015;89:867-82.

9. Guiu B, Assenat E. Doxorubicin for the treatment of hepatocellular carcinoma: GAME OVER! Ann Transl Med 2020;8:1693.

10. Li S, E M, Yu B. Adriamycin induces myocardium apoptosis through activation of nuclear factor $\mathrm{\kappa B}$ in rat. Mol Biol Rep 2008;35:489-94.

11. Sun J, Sun G, Meng X, et al. Isorhamnetin Protects against Doxorubicin-Induced Cardiotoxicity In Vivo and In Vitro. PLoS One 2013;8:e64526.

12. Wenningmann $N$, Knapp $M$, Ande A, et al. Insights into Doxorubicin-induced Cardiotoxicity: Molecular Mechanisms, Preventive Strategies, and Early Monitoring. Mol Pharmacol 2019;96:219-32.

13. Sahu BD, Kumar JM, Kuncha M, et al. Baicalein alleviates doxorubicin-induced cardiotoxicity via suppression of myocardial oxidative stress and apoptosis in mice. Life Sci 2016;144:8-18.

14. Murthy KNC, Jayaprakasha GK, Patil BS. Apoptosis mediated cytotoxicity of citrus obacunone in human pancreatic cancer cells. Toxicol in Vitro 2011;25:859-67.

15. Yao H, Shang Z, Wang $P$, et al. Protection of Luteolin7-O-Glucoside Against Doxorubicin-Induced Injury 
Through PTEN/Akt and ERK Pathway in H9c2 Cells. Cardiovasc Toxicol 2016;16:101-10.

16. Koleini N, Kardami E. Autophagy and mitophagy in the context of doxorubicin-induced cardiotoxicity. Oncotarget 2017;8:46663-80.

17. $\mathrm{Xu} \mathrm{HM}, \mathrm{Hu} \mathrm{F}$. The role of autophagy and mitophagy in cancers. Arch Physiol Biochem 2019;10:1-9.

18. Voigt N, Sadoshima J. Scientists on the Spot: Autophagy and heart disease. Cardiovasc Res 2019;115:e91-e92.

Cite this article as: Wang F, Wang L, Liu F, Meng L, Zhao N, Zhai X, Liu H, Yang J. Investigation of the mechanism of the reduction of anthracycline-induced cardiotoxicity by Qishen Huanwu Capsule based on network pharmacology. Ann Palliat Med 2021;10(1):16-28. doi: 10.21037/apm-20-2204
19. Zhou J, Yao W, Li C, et al. Administration of folliclestimulating hormone induces autophagy via upregulation of HIF- $1 \alpha$ in mouse granulosa cells. Cell Death Dis 2017;8:e3001.

20. Li Q, Yu Z, Xiao D, et al. Baicalein inhibits mitochondrial apoptosis induced by oxidative stress in cardiomyocytes by stabilizing MARCH5 expression. J Cell Mol Med 2020;24:2040-51. 